\title{
Extinction of goal tracking also eliminates the conditioned reinforcing effects of an appetitive conditioned stimulus
}

\author{
David N. Kearns • Brendan J. Tunstall • \\ Katherine R. Marks • Stanley J. Weiss
}

Published online: 17 November 2011

(C) Psychonomic Society, Inc. 2011

\begin{abstract}
Previous studies have suggested that the effects of extinction are response-specific. The present study investigated whether an extinction treatment that eliminated goal tracking elicited by an appetitive conditioned stimulus (CS) would also eliminate the conditioned reinforcing effects of that CS. Rats were first trained on a goal-tracking procedure in which an auditory CS was paired with a food unconditioned stimulus. Animals learned to approach the location where the food was delivered. In a subsequent phase, rats in one group received extinction training that eliminated the goal-tracking elicited by the CS. Rats in the other group did not experience extinction of the food-paired CS. Then, both groups received a test for conditioned reinforcement in which leverpresses resulted in the brief presentation of the stimulus previously paired with food. This stimulus did not act as a conditioned reinforcer in the group that had been subjected to extinction training, but did serve as a conditioned reinforcer in the group that did not experience extinction. These results indicate that the effects of extinction generalize from the approach-eliciting to the conditioned reinforcing effects of an appetitive CS.
\end{abstract}

Keywords Extinction · Conditioned reinforcement · Goal tracking $\cdot$ Rats

In extinction, a conditioned stimulus (CS; e.g., a tone) is presented without the unconditioned stimulus (US; e.g., food) with which the CS was previously paired. This typically results in a reduction in the frequency or magnitude of the conditioned response (CR; e.g., salivation) elicited by the CS. There are

D. N. Kearns $(\triangle) \cdot$ B. J. Tunstall $\cdot$ K. R. Marks $\cdot$ S. J. Weiss Psychology Department, American University,

4400 Massachusetts Ave NW,

Washington, DC 20016, USA

e-mail: kearns@american.edu several potential explanations for how extinction produces this decrease in conditioned responding. Perhaps the most intuitive of these is that extinction erases or weakens the CS-US association (Rescorla \& Wagner, 1972). Findings such as spontaneous recovery (e.g., Brooks \& Bouton, 1994), reinstatement (e.g., Rescorla \& Heth, 1975), and context renewal (e.g., Bouton \& Bolles, 1979) are problematic for this view, however, since these are all instances in which the $\mathrm{CR}$ reappears after extinction seems to have eliminated the CSUS association. An alternative view is that extinction involves the learning of a new inhibitory CS-US association (or CS-no US association), while the original CS-US association is preserved (Bouton, 1994, 2004; Pavlov, 1927). After extinction, presentation of the CS activates the inhibitory CS-US association (or CS-no US association), and therefore the CS does not elicit the CR. However, certain events (e.g., passage of time, change in context) can disrupt or prevent activation of this inhibitory association, and the intact original CS-US association is revealed through a reappearance of the CR.

A third view of how extinction reduces conditioned responding is that an inhibitory CS-CR association is learned during extinction. Several studies have supported this view by showing that the effects of extinction are response-specific (e.g., Bonardi, 1989; Delamater, 1996; Rescorla, 1993). For example, Delamater (Exp. 1, 1996) first trained rats on a procedure in which two CSs, a noise and a light, were each paired separately with a food or sucrose US. After repeated pairings, both CSs came to elicit a magazine approach (goaltracking) CR. Then, the CR elicited by one of the CSs was eliminated through extinction (i.e., the CS was presented without its US), while the other CS was not subjected to extinction. Surprisingly, there was no difference between the two stimuli on a transfer-of-control test in which each CS was superimposed on an operant baseline of leverpressing or chainpulling (for food or sucrose). That is, each CS was 
equally effective in facilitating operant responding, regardless of whether or not it had been previously extinguished. This and similar results led to the proposal that CS-US associations are preserved through extinction and the observed decrease in CR frequency is the result of an inhibitory stimulus-response association that is response-specific (Colwill, 1991; Delamater, 1996; Rescorla, 1993, 1996). The present experiment sought to determine whether a similar response specificity of extinction would be observed when an extinguished CS that had formerly elicited an appetitive CR was tested for its conditioned reinforcing properties.

Rats were first trained on a goal-tracking procedure in which an auditory CS was paired with a food US. After the goal-tracking response was established, rats were assigned to one of two groups. In a second phase, the extinction (Ext) group received extinction training (the $\mathrm{CS}$ was no longer paired with food) that eliminated goal tracking elicited by the CS. The no-extinction (No Ext) group did not experience extinction of this CS. Then a test of the conditioned reinforcing effects of the CS was administered to both groups. Evidence that extinction is response-specific would be provided if the previously food-paired CS served as an effective conditioned reinforcer in both groups.

\section{Method}

\section{Subjects}

A total of 20 naïve adult male Long-Evans rats served as subjects. The rats were deprived to approximately $80 \%$ of their free-feeding weights $(\sim 350-450 \mathrm{~g})$ throughout the experiment and were individually housed in stainless-steel hanging cages. They had free access to water and were fed approximately $15 \mathrm{~g}$ of laboratory rat chow following training sessions. The colony room where subjects were housed was on a 12:12 h light:dark cycle, with lights on at 08:00 $\mathrm{h}$. Training sessions were conducted during the light phase. Throughout the experiment, rats were treated in accordance with the Guide for the Care and Use of Laboratory Animals (National Academy of Sciences, 1996), as well as with the guidelines of American University's Institutional Animal Care and Use Committee.

\section{Apparatus}

Experimental sessions were conducted in five Coulbourn modular test cages $(30.0 \times 25.5 \times 29.0 \mathrm{~cm})$ whose front and rear walls and ceiling were aluminum. The two side walls were clear Plexiglas. Each chamber was equipped with two Coulbourn retractable levers located on the chamber's front wall. The left and right levers were positioned approximately $2 \mathrm{~cm}$ from the left and right side walls, respectively. Each lever measured $3.3 \mathrm{~cm}$ wide and, when inserted, extended approximately $1.5 \mathrm{~cm}$ into the chamber approximately $2.25 \mathrm{~cm}$ above the grid floor. Depressions of a lever in the extended state activated a microswitch that sent a $-28-\mathrm{V}$ DC input to the computer interface. Food was delivered via a Coulbourn food dispenser to a food receptacle (magazine) located inside an aperture in the front wall of the chamber between the two levers. A photobeam extended across this aperture approximately $0.5 \mathrm{~cm}$ inside the front chamber wall and was used to record magazine approach responses. Three Coulbourn stimulus lights that signaled the start and end of a session were located in the center of the front wall approximately $10 \mathrm{~cm}$ above the floor. A speaker connected to a Med Associates ANL 926 audio generator provided the tone $(4000-\mathrm{Hz}$, approximately $80-\mathrm{dB})$ and white noise (approximately 73-dB) stimuli. Experimental procedures were controlled by Med Associates software (Med-PC) running on a PC located in an adjacent room.

\section{Procedure}

Phase 1 Rats were first trained in a single session to eat from the food magazine on a procedure in which the food US was presented according to a variable-time (VT) 180-s schedule (range: 150-210 s). Each US consisted of three food pellets delivered in rapid succession ( $0.2 \mathrm{~s}$ apart). There were 12 US presentations over the course of an approximately 36-min session. In addition, five food pellets were placed in the food magazine prior to the start of the session. The levers remained in the retracted position.

Rats were then trained on a procedure in which one of the auditory CSs (the $\mathrm{CS}^{+}$) was paired with food. The other auditory CS (the $\mathrm{CS}^{-}$) served as a control stimulus and was not paired with food. The tone and the noise were counterbalanced over $\mathrm{CS}^{+} / \mathrm{CS}^{-}$designations. $\mathrm{CS}^{+}$trials lasted $15 \mathrm{~s}$ and terminated with the delivery of three food pellets. $\mathrm{CS}^{-}$ trials also lasted $15 \mathrm{~s}$ but did not end in food delivery. Intertrial intervals lasted $90 \mathrm{~s}$ on average (range: 60-120 s) and were timed from the offset of one CS to the onset of the next CS. $\mathrm{CS}^{+}$and $\mathrm{CS}^{-}$trials alternated randomly, with the restriction that there could be no more than two consecutive trials of the same type. Each CS was presented 18 times per session. Food magazine photobeam breaks were used to measure the goal-tracking CR. Photobeam breaks were recorded but had no programmed consequences at any time. Six sessions of this procedure were presented to each rat. The levers remained in the retracted position.

Phase 2 Rats were then assigned to either the Ext group or the No Ext group. Group assignments were made with the goal of matching groups on $\mathrm{CS}^{+}$and $\mathrm{CS}^{-}$goal-tracking response rates. The numbers of subjects in each group that had the tone as $\mathrm{CS}^{+}$and the noise as $\mathrm{CS}^{-}$, and vice versa, were 
approximately counterbalanced. (Due to an equipment malfunction, 2 rats had to be excluded from the study. This resulted in the numbers of rats trained with the tone vs. the noise as $\mathrm{CS}^{+}$being 5 and 4, respectively, for the No Ext group and 4 and 5, respectively, for the Ext group.) The Ext Group received seven sessions on an extinction procedure in which the $\mathrm{CS}^{+}$and $\mathrm{CS}^{-}$stimuli were presented without food. As previously, trials lasted $15 \mathrm{~s}$ and intertrial intervals lasted $90 \mathrm{~s}$ on average (range: $60-120 \mathrm{~s}$ ), and 18 trials of each type were presented per session. As before, goal-tracking CRs were recorded but had no consequences. The levers remained in the retracted position. The No Ext group was trained on the same procedure, except that $\mathrm{CS}^{+}$trials were omitted. That is, no stimulus was presented during those periods during which $\mathrm{CS}^{+}$was scheduled to occur. Thus, during Phase 2, rats in the No Ext Group experienced the same amount of exposure to the chamber and to $\mathrm{CS}^{-}$as did the Ext Group.

Conditioned reinforcement testing Rats in both groups then received three sessions to test for the conditioned reinforcing effects of the CSs. Both levers were inserted into the chambers for the duration these sessions. A single leverpress response on the left lever turned on the noise for $1 \mathrm{~s}$. A single press on the right lever turned on the tone for $1 \mathrm{~s}$. Food was not presented at any time during conditioned reinforcement sessions. The sessions lasted for $60 \mathrm{~min}$.

\section{Results}

\section{Phase 1}

The left-hand portion of Fig. 1 presents mean ( \pm SEM) goaltracking responses per session for the $\mathrm{CS}^{+}$and $\mathrm{CS}^{-}$during the six sessions of goal-tracking acquisition for both groups. As

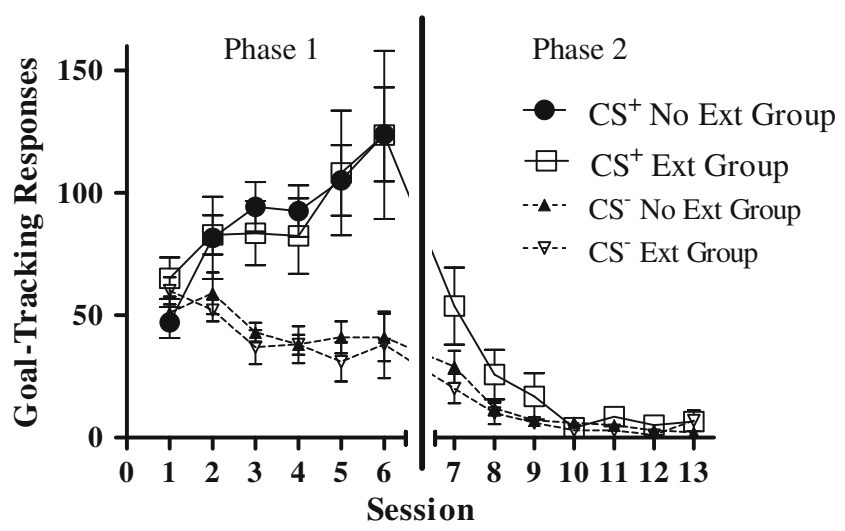

Fig. 1 The left portion of the figure presents mean $( \pm S E M)$ goaltracking responses per session for $\mathrm{CS}^{+}$and $\mathrm{CS}^{-}$for the No Ext and Ext groups during the conditioning phase. The right-hand portion presents mean $( \pm S E M) \mathrm{CS}^{+}$and $\mathrm{CS}^{-}$goal-tracking responses during Phase 2 the figure illustrates, rats' mean $\mathrm{CS}^{+}$responses rose to approximately 120 by the end of the phase, while $\mathrm{CS}^{-}$ responding was approximately one-third that amount. A $2 \times 6 \times 2$ (Stimulus $\times$ Session $\times$ Group) repeated measures ANOVA indicated a significant Stimulus $\times$ Session interaction $[F(5,80)=15.9, p<.001]$, as well as a significant effect of stimulus $[F(1,16)=35.7, p<.05]$, but there was no significant effect of group or any significant interactions involving the group variable (all $F_{\mathrm{s}}<1$ ).

Phase 2

The right-hand portion of Fig. 1 presents mean ( \pm SEM) goal-tracking responses during the seven sessions of Phase 2. Mean $\mathrm{CS}^{+}$responding declined significantly over sessions for the Ext group (the No Ext group did not experience $\mathrm{CS}^{+}$), and on the final session of this phase, rats made fewer than 10 total $\mathrm{CS}^{+}$responses. A repeated measures ANOVA performed on $\mathrm{CS}^{+}$responding confirmed that the effect of session was significant $[F(1,8)=$ $6.6, p<.05]$. A paired-samples $t$ test indicated that there was no difference between $\mathrm{CS}^{+}$and $\mathrm{CS}^{-}$responding for the Ext Group on the final session of the phase $[t(8)=0.1, p>.9]$.

\section{Conditioned reinforcement testing}

Figure 2 presents the mean $( \pm S E M)$ total number of leverpresses made on the levers associated with the $\mathrm{CS}^{+}$and $\mathrm{CS}^{-}$over the three conditioned reinforcement test sessions. Rats in the No Ext group made twice as many responses on the $\mathrm{CS}^{+}$lever as they did on the $\mathrm{CS}^{-}$lever. In contrast, rats in the Ext Group made similar numbers of responses on both levers. A $2 \times 2$ (Lever $\times$ Group) repeated measures ANOVA confirmed a significant Lever $\times$ Group interaction

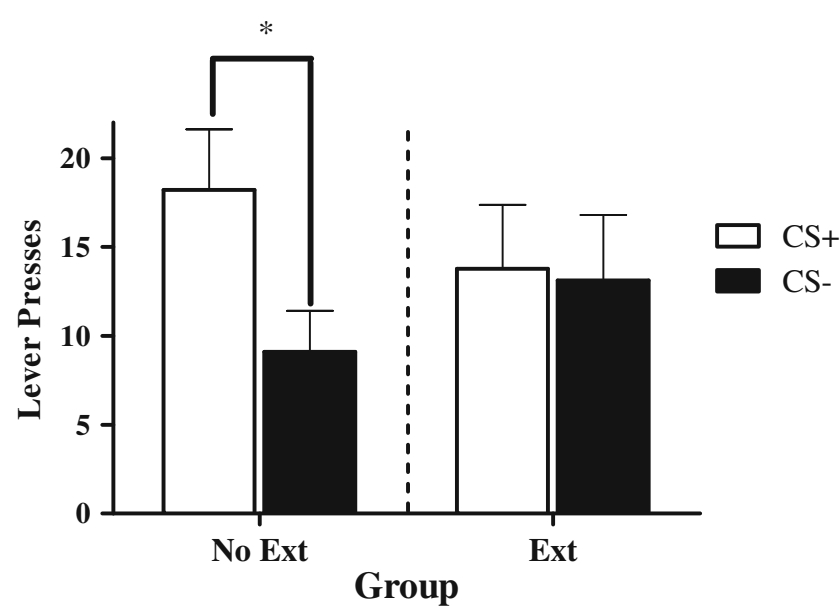

Fig. 2 Mean $( \pm S E M)$ total numbers of responses made during the three conditioned reinforcement test sessions for the No Ext and Ext groups. The white and black bars represent responding on the $\mathrm{CS}^{+}$and $\mathrm{CS}^{-}$levers, respectively. ${ }^{*} p<.005$ 
$[F(1,16)=5.1, p<.05]$. Subsequent paired-sample $t$ tests (Bonferroni corrected) indicated that rats in the No Ext Group responded significantly more on the $\mathrm{CS}^{+}$lever than on the $\mathrm{CS}^{-}$lever [t( 8$\left.)=3.9, p<.005\right]$, but there was no difference in responding over levers for the Ext group $[t(8)=0.2, p>.8]$.

\section{Discussion}

In the present experiment, rats in the Ext group received during Phase 2 an extinction treatment that eliminated goal tracking elicited by a stimulus $\left(\mathrm{CS}^{+}\right)$previously paired with food. On a subsequent test for conditioned reinforcement, this group did not press a lever to turn on this stimulus any more than they pressed a lever to turn on a control stimulus $\left(\mathrm{CS}^{-}\right)$that was never paired with food. In contrast, the No Ext group, that did not receive extinction of the food-paired stimulus during Phase 2, pressed a lever to turn on this stimulus at double the rate that they pressed a lever to turn on the control stimulus. These results indicate that the effects of extinction generalize from a goal-tracking CR elicited by a formerly food-paired CS to the conditioned reinforcing properties of that CS.

The present outcome contrasts with those of previous studies (e.g., Delamater, 1996) suggesting that extinction produces an inhibitory stimulus-response association that is specific to a particular response. Instead, the present results are consistent with the more common view that extinction decreases the net excitatory strength of the CS. This reduction in net excitation may occur through a direct weakening of the excitation conditioned to the CS (e.g., Rescorla \& Wagner, 1972) or through the learning of an inhibitory CS-US association in addition to the originally learned excitatory CS-US association (e.g., Bouton, 1994, 2004; Pavlov, 1927).

The idea that a response controlled by a CS will only be observed if the CS's net excitation surpasses a threshold (Weidemann \& Kehoe, 2003) can help to reconcile the results of the present experiment with those of the previous report that extinction of goal tracking elicited by a CS did not affect its transfer properties (Delamater, 1996). If the various behavioral effects produced by a CS require different thresholds of excitation, then at a given level of net excitation, some behavioral effects may be observed, while others are not. For example, extinction may reduce an appetitive CS's net excitation to a level that is below the threshold required to observe goal tracking and conditioned reinforcement, but not below the threshold required to observe transfer of control. (Evidence that instrumental transfer requires very little net excitation has come from previous experiments showing that the operant-rate-influencing effects of stimuli are retained even after very extensive extinction; Hendry, 1982; Kearns \& Weiss, 2005; Reberg, 1972). That different behavioral effects of a CS have different net excitation thresholds can explain why some studies (e.g., Delamater, 1996) have observed response-specific extinction and others (e.g., the present study) have found that extinction suppresses multiple behavioral effects of a CS.

Author Note This research was supported by Award Number R01DA008651 from the National Institute on Drug Abuse. The content is solely the responsibility of the authors and does not necessarily represent the official views of the National Institute on Drug Abuse or the National Institutes of Health.

\section{References}

Bonardi, C. (1989). Inhibitory stimulus control is specific to both the response and the reinforcer. Quarterly Journal of Experimental Psychology, 41B, 225-242.

Bouton, M. E. (1994). Conditioning, remembering, and forgetting. Journal of Experimental Psychology: Animal Behavior Processes, 20, 219-231.

Bouton, M. E. (2004). Context and behavioral processes in extinction. Learning \& Memory, 11, 485-494.

Bouton, M. E., \& Bolles, R. C. (1979). Role of conditioned contextual stimuli in reinstatement of extinguished fear. Journal of Experimental Psychology: Animal Behavior Processes, 5, 368-378.

Brooks, D. C., \& Bouton, M. E. (1994). A retrieval cue for extinction attenuates response recovery (renewal) caused by a return to the conditioning context. Journal of Experimental Psychology: Animal Behavior Processes, 20, 366-379.

Colwill, R. M. (1991). Negative discriminative stimuli provide information about the identity of omitted response-contingent outcomes. Animal Learning \& Behavior, 19, 326-336.

Delamater, A. (1996). Effects of several extinction treatments upon the integrity of Pavlovian stimulus-outcome associations. Animal Learning \& Behavior, 24, 437-449.

Hendry, J. S. (1982). Summation of undetected excitation following extinction of the CER. Animal Learning \& Behavior, 10, 476-482.

Kearns, D. N., \& Weiss, S. J. (2005). Reinstatement of a foodmaintained operant produced by compounding discriminative stimuli. Behavioural Processes, 70, 194-202.

National Academy of Sciences. (1996). Guide for the care and use of laboratory animals. Washington: National Academy Press.

Pavlov, I.P. (1927). Conditioned reflexes (G.V. Anrep, Trans.). London: Oxford University Press.

Reberg, D. (1972). Compound tests for excitation in early acquisition and after prolonged extinction of conditioned suppression. Learning and Motivation, 3, 246-258.

Rescorla, R. A. (1993). Inhibitory associations between S and R in extinction. Animal Learning \& Behavior, 21, 327-336.

Rescorla, R. A. (1996). Preservation of Pavlovian associations through extinction. Quarterly Journal of Experimental Psychology, 49B, 245-258

Rescorla, R. A., \& Heth, C. D. (1975). Reinstatement of fear to an extinguished conditioned stimulus. Journal of Experimental Psychology: Animal Behavior Processes, 1, 88-96.

Rescorla, R. A., \& Wagner, A. R. (1972). A theory of Pavlovian conditioning: Variations in the effectiveness of reinforcement and nonreinforcement. In A. H. Black \& W. F. Prokasy (Eds.), Classical conditioning II: Current research and theory (pp. 64-99). New York: Appleton-Century-Crofts.

Weidemann, G., \& Kehoe, E. J. (2003). Savings in classical conditioning in the rabbit as a function of extended extinction. Learning \& Behavior, 31, 49-68. doi:10.3758/BF03195970 